\title{
Update and Additional Frequencies for Kepler Star KIC 9700322
}

\author{
J. A. Guzik ${ }^{1} \&$ M. Breger ${ }^{2,3}$ \\ ${ }^{1}$ Los Alamos National Laboratory, XTD-2, MS T086, Los Alamos, NM 87545-2345, \\ USA \\ ${ }^{2}$ Institut für Astronomie der Universität Wien, Türkenschanzstr. 17, A-1180, Wien, \\ Austria \\ ${ }^{3}$ Department of Astronomy, University of Texas, Austin, TX 78712, USA
}

\begin{abstract}
Breger et al. (2011) reported on the discovery of 76 frequencies for the star KIC 9700322 from one month (Sept.-Oct. 2009) of short-cadence (1-minute sampling rate) photometric data during Quarter 3 of the NASA Kepler mission. Here we report on a reanalysis combining an additional month (June-July 2009) obtained during Quarter 2 as part of the Kepler Guest Observer program. This analysis confirms all but two of the earlier frequencies $>0.5 \mathrm{c} / \mathrm{d}$, and in addition finds six new combination frequencies of the 8 highest amplitude modes, and three frequencies that are not combinations of these modes. Since we do not know the parents of 12 of the 83 frequencies, additional astrophysical discoveries may await in continued study of this star. The rotational modulations of the two radial modes and the $\ell=2$ quintuplet are confirmed. For the two radial modes $\left(f_{1}, f_{2}\right)$ and the central $(m=0)$ mode of the $\ell=2$ quintuplet, $f_{6}$, amplitude variability and/or close frequencies were found. The additional data do not reveal more of the $\ell=0,1,2$ or higher-degree $\mathrm{p}$ modes that were expected, and this result is still a mystery.
\end{abstract}

Accepted: June 27, 2011

Individual Objects: KIC 9700322

\section{Introduction}

As part of its search for Earth-sized planets around sun-like stars, the Kepler mission is surveying around 150,000 stars for planetary transits. In the process, 
it is acquiring photometry with unprecedented precision and continuous length of time series on hundreds of pulsating variable stars, including many $\delta$ Scuti stars (Borucki et al. 2010; Gilliland et al. 2010).

The $\delta$ Scuti star KIC 9700322 is a slowly rotating, cool $\delta$ Scuti star $\left(\mathrm{T}_{\text {eff }}=\right.$ $6700 \pm 100 \mathrm{~K}, \log g=3.7 \pm 0.1, v \sin i=19 \pm 1 \mathrm{~km} / \mathrm{s}$; Breger et al. 2011). $\delta$ Sct stars are variable stars of A-F spectral type on or near the main sequence that show radial and nonradial oscillations with frequencies $\sim 5-30$ cycles/day. The pulsations are driven by the " $\kappa$ effect", a feedback mechanism produced by the opacity increase in the region of second helium ionization in the stellar envelope (see, e.g., Aerts et al. 2010). The brightness variations of KIC 9700322 were measured by the Kepler satellite for 30.3 d during Quarter 3. Analyses of these data revealed 76 frequencies with amplitudes as small as 14 ppm. Two dominant radial modes, an $\ell=2$ quintuplet and a brightness modulation during rotation were detected (i.e., eight frequencies). Almost all other detected frequencies could be identified with various combinations and rotational modulations of these modes. Good agreement of the frequency spacings with theoretical models could be obtained (Breger et al. 2011, hereafter called Paper I). A remarkable result was the absence of additional independent frequencies down to an amplitude limit near $14 \mathrm{ppm}$, suggesting that the star is stable against most forms of pulsation.

In 2008, as part of the Kepler Guest Observer Cycle 1 program, before Kepler was launched, J.A. Guzik proposed to observe KIC 9700322 along with thirteen other stars in a search for hybrid $\gamma$ Doradus $/ \delta$ Sct stars, and the data was taken in Q2. Because KIC 9700322 did not show hybrid behavior with both higher (10-15 c/d) and low (1-5 c/d) frequencies, this star was not considered further until the Paper I preprint appeared. Since we realized that we had additional short cadence data readily available, we decided to combine the two quarters of data to see whether the results from one quarter could be confirmed and extended.

\section{The Q2 Kepler spacecraft observations of KIC 9700322}

Portions of the Q2 "raw" data (that have been processed to reduce the effects of bias and dark current, and flat-fielded) have small systematic zero-point drifts in brightness. These shifts are removed in the "corrected" data provided by the Kepler team, but these data that have been pre-conditioned to optimize the search for planetary transits (see http://keplergo.arc.nasa.gov/PipelinePDC.shtml) are not suitable for asteroseismological investigations, and so were not used here. In the Q2 data for KIC9700322, a two-day stretch immediately after an observing gap near HJD 2455016 contains a large drift reaching several parts per thousand. These 


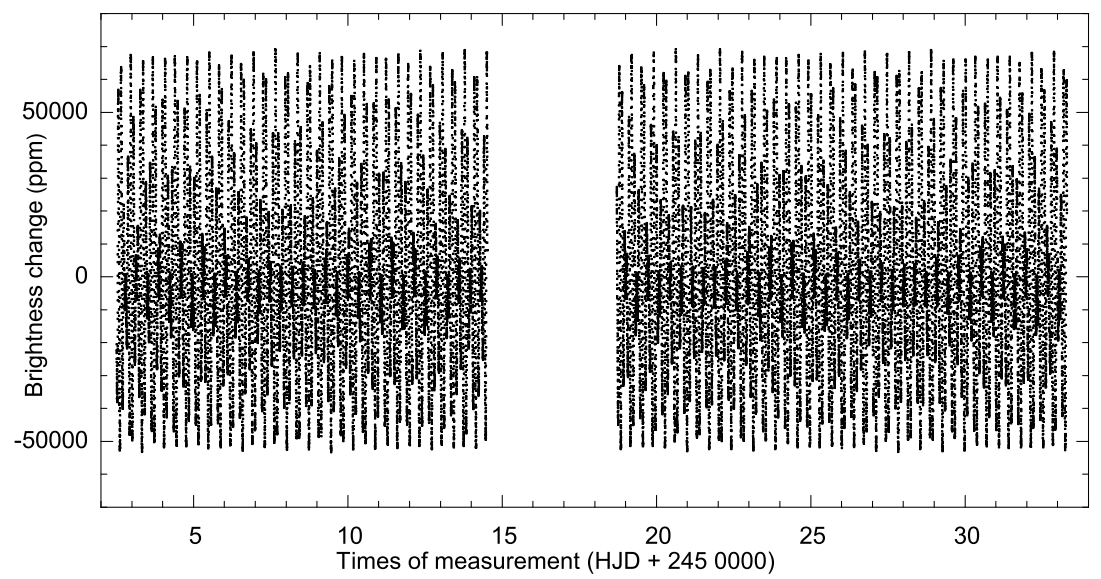

Figure 1: Light curve of KIC9700322 after reductions of the new Q2 Kepler data.

data, from HJD 2455016.71 to 245 5018.71, were not used. For the remaining data, the small remaining zero-point drifts were removed artificially, similar to the techniques applied by Garcia et al. (2011). This processing affects only the analysis of the low-frequency region. Consequently, the present paper covers only periods shorter than two days, or $f>0.5 \mathrm{c} / \mathrm{d}$, i.e., the period range of the typical $\delta$ Sct stars. As a final step, the data were filtered by us for obvious outliers (deviations larger than $4 \sigma$ ). Fig. 1 shows the new observations after our reductions.

\section{Frequency analysis}

The KIC 970032 Kepler data were analyzed with the statistical package PERIOD04 (Lenz \& Breger 2005). This package carries out multifrequency analyses with Fourier as well as least-squares algorithms. White noise is not assumed. Following the standard procedures for examining the peaks with PERIOD04, we have determined the amplitude signal/noise values for every promising peak in the amplitude spectrum and adopted a limit of $\mathrm{S} / \mathrm{N}$ of 4.0. As in the previous paper on this star, the noise is calculated from prewhitened data because of the huge range in amplitudes of three orders of magnitude.

It was found that the Q2 data essentially confirms (and extends) the results previously obtained from the Q3 data alone. The amplitude spectra of Q2 and Q3 are essentially identical to each other (except for alias structure in Q2 
because of the gap in the middle of Q2). This demonstrates the stability of the pulsation of KIC 9700322. The matching of the Q2 and Q3 data also shows that the quality of the data in the two observing quarters is comparable, despite the more significant instrumental systematics in the Q2 data. Due to the similarity, new figures of the amplitude spectra are not shown in this paper and we refer to the figures in Paper I. We will now concentrate on the analysis of the combined Q2 and Q3 data, which covers 121d with a central gap of 60d. The long time base improves the frequency resolution and allows us to look for amplitude variability and close frequencies. Also, the additional data allow us to detect several additional frequencies.

79 statistically significant frequency peaks were detected in the frequency range $>0.5$ cycle $c / d$. These frequencies are listed in Table 1 , along with four frequencies $<0.5 \mathrm{c} / \mathrm{d}$ that were reported using the Q3 data in Paper 1 . Since the rotational frequency, $f_{3}=0.1597 \mathrm{c} / \mathrm{d}$, is important for modulations of the amplitudes of most (all?) pulsation modes, the rotational frequency and its multiples are also listed in the table with the values taken from Paper I.

Of the 9 new frequencies detected in the combined Q2Q3 analysis, 6 are easily identified as combination modes of the main frequencies. One frequency forms a close double with $f_{6}$ (see below). On the other hand, the smallamplitude peak from Paper I at $51.752 \mathrm{c} / \mathrm{d}$ is no longer statistically significant, while the unconfirmed peak at $12.584 \mathrm{c} / \mathrm{d}$ probably arose from the slow amplitude modulation of $f_{2}$ (see below).

71 of the 83 frequencies listed in Table 1 can be identified with $f_{1}-f_{8}$ and their combinations. Only 12 frequencies cannot be identified in this manner. However, these 12 frequencies are not all additional, independent pulsation modes. It is interesting that most of these frequencies are numerically related to each other through new combinations, e.g., the difference between the two peaks at 12.5333 and $22.3258 \mathrm{c} / \mathrm{d}$ is exactly $f_{1}$. Since $12.5333 \mathrm{c} / \mathrm{d}$ is a close double to $f_{2}, 22.3258 \mathrm{c} / \mathrm{d}$ is the identical close double to the $\left(f_{1}+f_{2}\right)$ peak.

Table 1: Multifrequency solution of KIC 9700322 and identifications.

\begin{tabular}{|c|c|c|c|c|c|c|}
\hline \multirow{2}{*}{$\begin{array}{c}\text { Frequency } \\
\qquad \mathrm{c} / \mathrm{d}\end{array}$} & \multirow[b]{2}{*}{$\mu \mathrm{Hz}$} & \multirow[t]{2}{*}{ Identification } & \multicolumn{3}{|c|}{ Amplitudes in ppm } & \multirow[t]{2}{*}{ Comment } \\
\hline & & & Q2Q3 & Q2 & Q3 & \\
\hline \multicolumn{7}{|c|}{ Main frequencies } \\
\hline 9.7925 & 113.339 & $f_{1}$ & 27302 & 27343 & 27266 & Radial \\
\hline 12.5688 & 145.472 & $f_{2}$ & 29449 & 29463 & 29440 & Radial \\
\hline 0.1597 & 1.848 & $f_{3}$ & & & 80 & Rotation \\
\hline 11.3187 & 131.003 & $f_{4}$ & 27 & 25 & 29 & Quintuplet \\
\hline 11.4551 & 132.582 & $f_{5}$ & 147 & 147 & 147 & Quintuplet \\
\hline
\end{tabular}

Continued... 
...continued from previous page

\begin{tabular}{|c|c|c|c|c|c|c|}
\hline \multirow{2}{*}{$\begin{array}{c}\text { Frequency } \\
\text { c/d }\end{array}$} & \multirow{2}{*}{\multicolumn{2}{|c|}{$\mu \mathrm{Hz}$}} & \multicolumn{3}{|c|}{ Amplitudes in ppm } & \multirow[t]{2}{*}{ Comment } \\
\hline & & & Q2Q3 & Q2 & Q3 & \\
\hline 11.5906 & 134.150 & $f_{6}$ & 465 & 474 & 460 & Quintuplet \\
\hline 11.7201 & 135.649 & $f_{7}$ & 215 & 207 & 222 & Quintuplet \\
\hline 11.8576 & 137.241 & $f_{8}$ & 115 & 118 & 112 & Quintuplet \\
\hline \multicolumn{7}{|c|}{ Combination frequencies } \\
\hline 0.3194 & 3.697 & $2 f_{3}$ & & & 174 & $2 \times$ rotation \\
\hline 0.4791 & 5.545 & $3 f_{3}$ & & & 33 & $3 \times$ rotation \\
\hline 0.6388 & 7.394 & $4 f_{3}$ & 17 & 13 & 25 & \\
\hline 0.7112 & 8.232 & $f_{2}-f_{8}$ & 16 & 19 & 26 & \\
\hline 0.9782 & 11.322 & $f_{2}-f_{6}$ & 31 & 36 & 26 & \\
\hline 1.1137 & 12.890 & $f_{2}-f_{5}$ & 48 & 55 & 46 & \\
\hline 1.6625 & 19.242 & $f_{5}-f_{1}$ & 20 & 16 & 25 & \\
\hline 1.7980 & 20.811 & $f_{6}-f_{1}$ & 27 & 39 & 17 & \\
\hline 2.7763 & 32.133 & $f_{2}-f_{1}$ & 2640 & 2647 & 2633 & \\
\hline 2.9360 & 33.981 & $f_{2}-f_{1}+f_{3}$ & 22 & 18 & 26 & \\
\hline 5.5526 & 64.266 & $2 f_{2}-2 f_{1}$ & 86 & 84 & 89 & \\
\hline 7.0162 & 81.206 & $2 f_{1}-f_{2}$ & 630 & 628 & 632 & \\
\hline 9.4731 & 109.643 & $f_{1}-2 f_{3}$ & 50 & 47 & 58 & \\
\hline 9.6328 & 111.491 & $f_{1}-f_{3}$ & 52 & 54 & 51 & \\
\hline 9.9522 & 115.188 & $f_{1}+f_{3}$ & 57 & 63 & 53 & \\
\hline 10.1119 & 117.036 & $f_{1}+2 f_{3}$ & 39 & 37 & 38 & \\
\hline 12.2494 & 141.776 & $f_{2}-2 f_{3}$ & 34 & 35 & 35 & \\
\hline 12.4091 & 143.624 & $f_{2}-f_{3}$ & 36 & 37 & 32 & \\
\hline 12.7285 & 147.321 & $f_{2}+f_{3}$ & 33 & 34 & 32 & \\
\hline 12.8882 & 149.169 & $f_{2}+2 f_{3}$ & 21 & 18 & 23 & \\
\hline 15.3451 & 177.605 & $2 f_{2}-f_{1}$ & 497 & 492 & 503 & \\
\hline 16.8088 & 194.546 & $3 f_{1}-f_{2}$ & 67 & 64 & 70 & \\
\hline 18.1214 & 209.738 & $3 f_{2}-2 f_{1}$ & 14 & 16 & 11 & \\
\hline 19.5850 & 226.679 & $2 f_{1}$ & 2226 & 2227 & 2225 & \\
\hline 21.2476 & 245.921 & $f_{5}+f_{1}$ & 45 & 48 & 42 & \\
\hline 21.3831 & 247.489 & $f_{6}+f_{1}$ & 40 & 46 & 36 & \\
\hline 21.5126 & 248.988 & $f_{7}+f_{1}$ & 18 & 22 & 16 & \\
\hline 21.6501 & 250.580 & $f_{8}+f_{1}$ & 18 & 17 & 19 & \\
\hline 22.2016 & 256.963 & $f_{1}+f_{2}-f_{3}$ & 26 & 26 & 26 & \\
\hline 22.3613 & 258.812 & $f_{1}+f_{2}$ & 4900 & 4899 & 4901 & \\
\hline 22.5210 & 260.660 & $f_{1}+f_{2}+f_{3}$ & 21 & 25 & 18 & \\
\hline 22.7738 & 263.585 & $f_{4}+f_{5}$ & 13 & 11 & 15 & \\
\hline
\end{tabular}


...continued from previous page

\begin{tabular}{|c|c|c|c|c|c|c|}
\hline \multirow{2}{*}{$\begin{array}{c}\text { Frequency } \\
\text { c/d }\end{array}$} & \multirow[b]{2}{*}{$\mu \mathrm{Hz}$} & \multirow[t]{2}{*}{ ID } & \multicolumn{3}{|c|}{ Amplitudes in $\mathrm{ppm}$} & \multirow[t]{2}{*}{ Comment } \\
\hline & & & Q2Q3 & Q2 & Q3 & \\
\hline 23.0456 & 266.732 & $f_{5}+f_{6}$ & 34 & 31 & 39 & \\
\hline 23.1811 & 268.300 & $2 f_{6}$ & 10 & 15 & 6 & \\
\hline 23.7152 & 274.481 & $2 f_{8}$ & 25 & 21 & 28 & \\
\hline 24.0239 & 278.054 & $f_{5}+f_{2}$ & 34 & 34 & 34 & \\
\hline 24.1594 & 279.622 & $f_{6}+f_{2}$ & 47 & 48 & 46 & \\
\hline 24.2889 & 281.121 & $f_{7}+f_{2}$ & 52 & 51 & 52 & \\
\hline 24.4264 & 282.713 & $f_{8}+f_{2}$ & 54 & 52 & 56 & \\
\hline 24.9779 & 289.096 & $2 f_{2}-f_{3}$ & 17 & 21 & 16 & \\
\hline 25.1376 & 290.945 & $2 f_{2}$ & 2661 & 2658 & 2663 & \\
\hline 26.6013 & 307.885 & $4 f_{1}-f_{2}$ & 13 & 14 & 12 & \\
\hline 27.9139 & 323.078 & $3 f_{2}-f_{1}$ & 203 & 203 & 203 & \\
\hline 29.3776 & 340.018 & $3 f_{1}$ & 190 & 188 & 191 & \\
\hline 32.1538 & 372.151 & $2 f_{1}+f_{2}$ & 482 & 485 & 479 & \\
\hline 33.8164 & 391.393 & $f_{1}+f_{2}+f_{5}$ & 12 & 15 & 10 & \\
\hline 33.9519 & 392.962 & $f_{1}+f_{2}+f_{6}$ & 15 & 14 & 16 & \\
\hline 34.2189 & 396.052 & $f_{1}+f_{2}+f_{8}$ & 17 & 19 & 16 & \\
\hline 34.9301 & 404.284 & $f_{1}+2 f_{2}$ & 536 & 536 & 536 & \\
\hline 37.7064 & 436.417 & $3 f_{2}$ & 329 & 329 & 329 & \\
\hline 39.1701 & 453.357 & $4 f_{1}$ & 19 & 23 & 16 & \\
\hline 40.4827 & 468.550 & $4 f_{2}-f_{1}$ & 34 & 34 & 34 & \\
\hline 41.9464 & 485.490 & $3 f_{1}+f_{2}$ & 22 & 22 & 23 & \\
\hline 44.7227 & 517.623 & $2 f_{1}+2 f_{2}$ & 113 & 112 & 114 & \\
\hline 47.4989 & 549.756 & $f_{1}+3 f_{2}$ & 78 & 74 & 82 & \\
\hline 50.2752 & 581.889 & $4 f_{2}$ & 82 & 80 & 83 & \\
\hline 51.7389 & 598.830 & $4 f_{1}+f_{2}$ & 9 & 8 & 10 & \\
\hline 54.5152 & 630.963 & $3 f_{1}+2 f_{2}$ & 35 & 34 & 35 & \\
\hline 57.2915 & 663.096 & $2 f_{1}+3 f_{2}$ & 55 & 53 & 58 & \\
\hline 60.0677 & 695.229 & $f_{1}+4 f_{2}$ & 34 & 34 & 34 & \\
\hline 62.8440 & 727.361 & $5 f_{2}$ & 18 & 21 & 15 & \\
\hline 67.0840 & 776.435 & $3 f_{1}+3 f_{2}$ & 19 & 18 & 19 & \\
\hline 69.8603 & 808.568 & $2 f_{1}+4 f_{2}$ & 19 & 16 & 21 & \\
\hline \multicolumn{7}{|c|}{ Peaks detected in Q3 with frequency $<0.5 \mathrm{c} / \mathrm{d}$} \\
\hline 0.0221 & 0.256 & $f_{72}$ & & & 347 & \\
\hline 0.0555 & 0.642 & $f_{73}$ & & & 95 & \\
\hline 0.1346 & 1.558 & $f_{74}$ & & & 35 & \\
\hline 0.3542 & 4.100 & $f_{75}$ & & & 25 & \\
\hline
\end{tabular}

Continued... 
..continued from previous page

\begin{tabular}{rrrrrrr}
\hline $\begin{array}{c}\text { Frequency } \\
\text { c/d }\end{array}$ & $\mu \mathrm{Hz}$ & ID & Qmplitudes in ppm & Comment \\
\hline \multicolumn{7}{c}{ Other peaks in the amplitude spectrum } \\
8.1394 & 94.206 & $f_{76}$ & 15 & 11 & 18 & \\
11.5806 & 134.035 & $f_{77}$ & 135 & 140 & 132 & \\
11.9712 & 138.555 & $f_{78}$ & 16 & 19 & 13 \\
12.5333 & 145.061 & $f_{79}$ & 61 & 37 & 80 \\
13.2406 & 153.247 & $f_{80}$ & 18 & 16 & 21 \\
14.6253 & 169.274 & $f_{81}$ & 14 & 10 & 17 \\
22.3258 & 258.400 & $f_{82}$ & 15 & 8 & 20 \\
24.1493 & 279.506 & $f_{83}$ & 45 & 48 & 42 & \\
\hline
\end{tabular}

\section{Amplitude variability/close frequencies}

Amplitude variability and the presence of close-frequency pairs (which lead to amplitude variability in short data sets) were examined in two ways. To examine the amplitude variability directly, we have subdivided the data into three-day groups. For each group we have computed the optimum amplitudes for the dominant modes, while using a common solution for the other frequencies. The second method relied on Fourier analyses and multiple-least-squares solutions in PERIOD04 to look for close frequencies.

For the two radial modes $\left(f_{1}, f_{2}\right)$ and the central $(m=0)$ mode of the $\ell=$ 2 quintuplet, $f_{6}$, amplitude variability and/or close frequencies were found, that we discuss below.

\section{1. $f_{6}=11.591 \mathrm{c} / \mathrm{d}$}

In the previous paper we identified $f_{6}$ as the axisymmetric mode $(m=0)$ of an $\ell=2$ quintuplet. When we add the Q2 data, it becomes obvious that the mode has a variable amplitude, possibly caused by a close companion frequency. The amplitude variability is shown in Fig. 2. If we assume a sinusoidal amplitude variation, we obtain a modulation frequency of $0.010 \pm 0.001 \mathrm{c} / \mathrm{d}$ or a modulation period near $100 \mathrm{~d}$.

This $100 \mathrm{~d}$ modulation should also be visible in the Fourier spectrum. In the individual Q2 or Q3 data sets alone, the frequency resolution is not sufficient to reveal the double structure. When the two data sets are combined, the double frequency structure becomes apparent. Some uncertainties due to aliasing (structures separated by $0.011 \mathrm{c} / \mathrm{d}$ ) still remain. When we prewhiten 


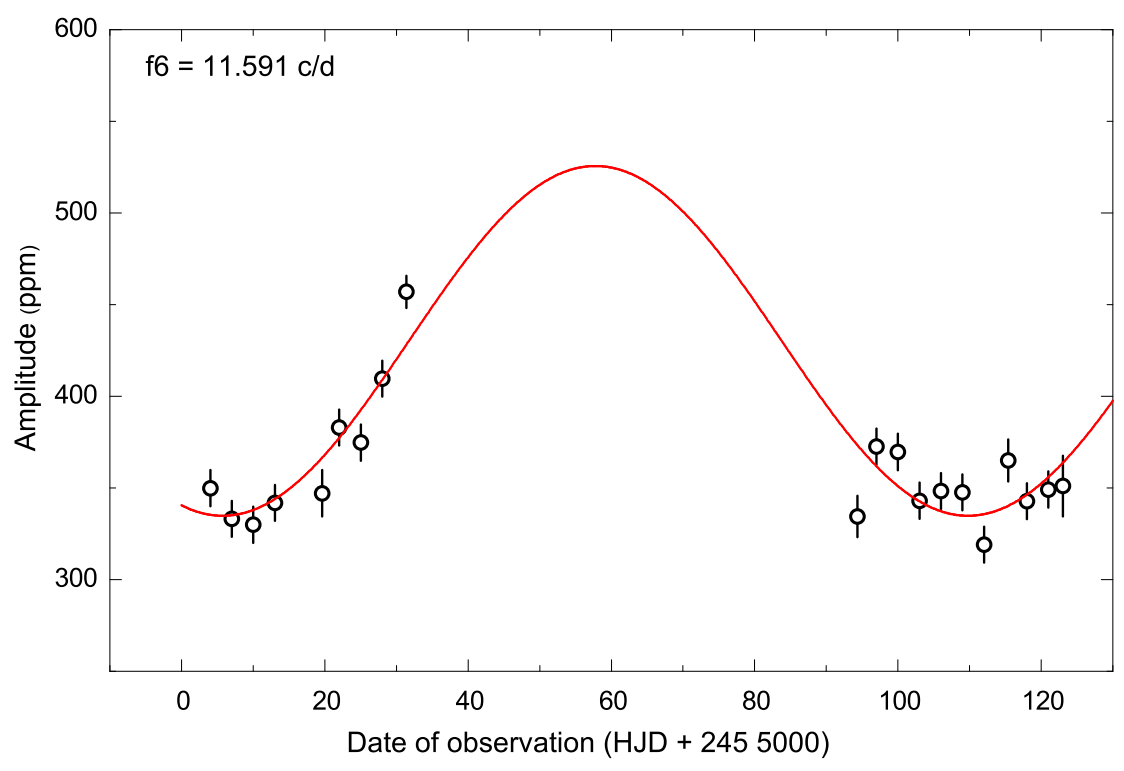

Figure 2: Amplitude variation of $f_{6}$. This is interpreted as beating between two close frequencies.

the dominant frequency of $11.591 \mathrm{c} / \mathrm{d}$, a number of peaks remain in the amplitude spectrum. The peak at $11.581 \mathrm{c} / \mathrm{d}$ for the second peak leads to a clean power spectrum after prewhitening. An alias peak at $11.569 \mathrm{c} / \mathrm{d}$, on the other hand, is unsatisfactory because it leaves a number additional close peaks and leads to higher residuals. We, therefore, adopt $11.581 \mathrm{c} / \mathrm{d}$ for the second peak. Our adopted frequency difference between the two frequencies in the doublet is in agreement with the value derived above from inspecting the amplitude variation.

Furthermore, the fact that we obtain only two statistically significant peaks (as opposed to a triplet with the main frequency in the middle) in the power spectrum supports the interpretation as beating between two close frequencies, rather than plain amplitude variability. Nevertheless, we must caution against potential overinterpretation of our data, since the length of data set is similar to the detected modulation period.

\subsection{Radial mode $f_{1}$}

We have examined potential amplitude variation in the same manner as for $f_{6}$. The radial mode $f_{1}$ varies by about $0.2 \%$ of its value with a period of about $125 \mathrm{~d}\left(f_{\bmod }=0.0081 \pm 0.004 \mathrm{c} / \mathrm{d}\right)$. Since the modulation amplitude is very 
small and the modulation period is similar to the length of the data set, Fourier analyses of this modulation remain inconclusive.

\subsection{Radial mode $f_{2}$}

In the Q3 data alone we detected a second frequency, separated from $f_{2}$ by about $0.03 \mathrm{c} / \mathrm{d}$. We confirm the existence of this frequency, for which an improved value of $12.533 \mathrm{c} / \mathrm{d}$ is determined. The amplitudes of $f_{2}$ suggest that an additional small amplitude modulation of $f_{2}$ with a time scale in excess of $150 \mathrm{~d}$ also exists. Due to the small size of this variation we cannot obtain a more quantitative result at this stage.

\section{Discussion}

KIC 9700322 is remarkably stable compared to some $\delta$ Sct stars that show larger frequency amplitude variations over periods of weeks (see e.g., Breger 2009 and discussion on the $\delta$ Sct star 4 CVn.) KIC 9700322 shares with other stars with amplitude variability a long-term modulation of hundreds of days. Understanding the reasons for these amplitude and frequency variations requires long-term monitoring and high photometric precision. There are other mysteries associated with KIC 9700322 that are also present for other $\delta$ Sct stars: Why, for example, does this star show only a couple of radial modes and one $\ell=2 \mathrm{p}$-mode, and not other expected low-degree modes? Which (invisible?) frequencies are the parents of the new pulsation modes that are not combinations of the modes of highest amplitude? Why does this star not show any lower-frequency $\gamma$ Dor pulsations as do most of the $A$ and early $F$ main sequence stars observed by Kepler (Grigahcéne et al. 2010; Balona et al. 2011)? KIC 9700322 continues to be monitored, and analysis of an additional 3 months of short cadence data from Q6 will begin soon. In addition, long-cadence (29.4-minute integration time) data exist for Q0 through Q7, so future work may be able to explore longer-term trends. Hopefully, an analysis of KIC 9700322 in comparison and contrast with the many other $\gamma$ Dor and $\delta$ Sct stars being observed by Kepler will help to solve some of the puzzles associated with the pulsation behavior of these stars.

Acknowledgments. This investigation has been supported by the Austrian Fonds zur Förderung der wissenschaftlichen Forschung through project P 21830N16. We thank the entire Kepler team for making this data possible. J.G. is pleased to acknowledge support and data acquisition from the Kepler Guest Observer Cycle 1 program grant number KEPLER08-0013. 


\section{References}

Aerts, C., Christensen-Dalsgaard, J. \& Kurtz, D. W. 2011, Asteroseismology, Astronomy and Astrophysics Library, Springer, pp. 49-56

Balona, L., Guzik, J. A., Uytterhoeven, K., et al. 2011, "The Kepler View of $\gamma$ Dor Stars", MNRAS, in press

Borucki, W. J. , Koch, D., Basri, G., et al. 2010, Science, 327, 977

Breger, M., Balona, L., Lenz, P., et al. 2011, MNRAS, 414 doi: 10.1111/j.1365-2966.2011.18508 (Paper I)

Breger, M. 2009, "Period Variations in $\delta$ Scuti Stars," in Stellar Pulsation: Challenges for Theory and Observation, eds. J.A. Guzik and P.A. Bradley, AIPC conference proceedings Volume 1170, pp. 410-414

Garcia, R. A., Hekker, S., Stello, D. et al. 2011, MNRAS, 414, L6

Gilliland, R. L., Brown, T. M., Christensen-Dalsgaard, J., et al. 2010, PASP, 122, 131

Grigahcéne, A., Antoci, V., Balona, L., et al. 2010, ApJ, 713, 192

Lenz, P. \& Breger, M. 2005, CoAst, 146, 53 
